\title{
Genetic enhancement in cultured human adult stem cells conferred by a single nucleotide recoding
}

Cell Research (2017) 27:1178-1181. doi:10.1038/cr.2017.86; published online 7 July 2017

\section{Dear Editor,}

Cell replacement therapy holds great therapeutic promise towards restoring tissue homeostasis. However, aging-associated functional decay and neoplastic transformation following transplantation must be overcome before cell therapies can be effectively and safely implemented in the clinical settings [1-4]. This goal can theoretically be achieved by developing enhancement strategies towards endowing stem cells with more robust regenerative capacities and concomitantly, a reduced risk for tumorigenesis $[5,6]$. Here, we provide an experimental and conceptual strategy on how to genetically engineer superior and safer stem cells.

We first aimed to identify and manipulate pathways or key genes able to repress cellular aging. To this end, we screened several gero-protective chemicals using Werner Syndrome (WS, WRN-deficient) human mesenchymal stem cell (hMSC) aging model [7]. We found Oltipraz, Metformin, and Resveratrol at indicated concentrations stimulated the proliferation of pre-senescent WS hMSCs (Supplementary information, Figure S1A). Interestingly, these three chemicals are all known activators of the nuclear factor erythroid-2-like 2 (NRF2) pathway (Supplementary information, Figure S1B). In addition, the protein abundance of NRF2 was decreased in WS or replicative senescent hMSCs (Supplementary information, Figure S1C). These raise the possibility of enhancing NRF2 activity to augment the function and lifespan of hMSCs.

To activate NRF2 in a stable and controllable manner while retaining NRF2's native biological function with minimal genomic change, we focused on generating a single-nucleotide variation (A245G) in the $N R F 2$ locus, which results in a glutamic acid to glycine switch at amino acid 82 of the NRF2 protein, and is supposed to lead to NRF2 stabilization and transcriptional activation of its target genes. We performed homologous recombination-based gene editing using helper-dependent adenoviral vector (HDAdV) [7] in WT human embryonic stem cells (ESCs) $\left(\mathrm{NRF}^{+/+} \mathrm{hESCs}\right)$ and generated homozygous $N R F 2^{A 245 G}$ ESC lines (NRF2 $2^{A G / A G} \mathrm{hESCs}$ ) (Figure 1A and Supplementary information, Figure S2A and S2B). $N R F 2^{A G / A G}$ hESCs exhibited features of typical human primed state pluripotent stem cells (Supplementary information, Figure S2C and S2D) and were subsequently differentiated into hMSCs (Supplementary information, Figure S3A and S3B). In $N R F 2^{A G / A G}$ hMSCs, we found the endogenous NRF2 protein level was upregulated with more enrichment in the nucleus, which was accompanied by elevated NRF2 transcriptional activity (Figure $1 \mathrm{~B}$ and Supplementary information, Figure S3C-S3F). Importantly, genomic integrity was preserved after gene editing and differentiation procedures (Supplementary information, Figure $\mathrm{S} 3 \mathrm{G}$ and $\mathrm{S} 3 \mathrm{H}$ ).

We next tested whether NRF2 enhancement is sufficient to counteract cellular senescence. Upon serial passaging, $N R F 2^{+/+}$hMSCs ceased growth after passage 10 (P10), whereas $N R F 2^{A G / A G}$ hMSCs maintained a robust proliferation until P19 (Figure 1C and Supplementary information, Figure S4A and S4B). In contrast to $N R F 2^{+/+}$ hMSCs, which exhibited progressive senescence, significantly lower percentages of senescence-associated (SA)- $\beta$-gal-positive cells and lower expression of senescence-associated genes $\left(p 16^{\text {Ink4a }}\right.$ and $\left.p 21^{\text {Wafl }}\right)$ were observed in $N R F 2^{A G / A G}$ hMSCs (Figure 1D and Supplementary information, Figure S4C-S4E).

In line with NRF2's role in anti-oxidation, we observed lower basal ROS levels and fewer oxidative byproducts in $N R F 2^{A G / A G}$ hMSCs even after extensive passaging (Supplementary information, Figure S4F-S4H). Lowered ROS levels conferred by engineered NRF2 also alleviated senescence-associated defects in the nuclear envelope (NE) and in the (epi-)genome (Supplementary information, Figure S5). Similarly, human vascular endothelial cells (hVECs) [8] derived from $N R F 2^{A G / A G} \mathrm{hESCs}$ exhibited substantial resistance to oxidative stress (Supplementary information, Figure S6). Together, these observations indicate that genetic activation of endogenous NRF2 helps alleviate aging-associated oxidative stress and subsequent cellular damage.

Moreover, we demonstrated this gene-editing strategy could elicit a therapeutic effect in the WS background. Genetic enhancement of NRF2 in WS hMSCs increased 
A
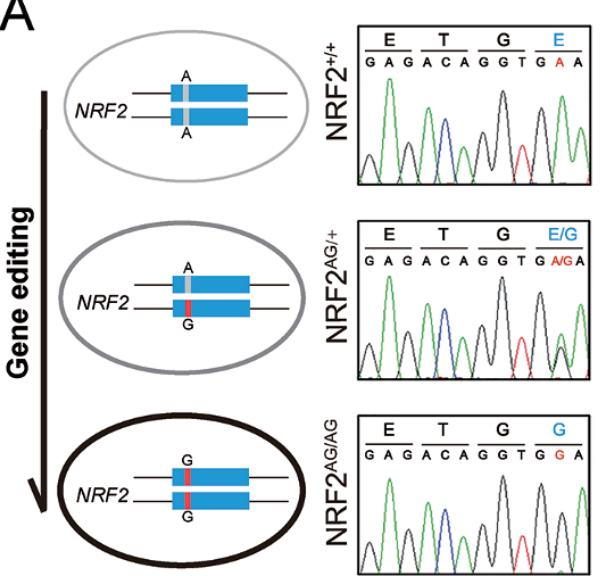

C

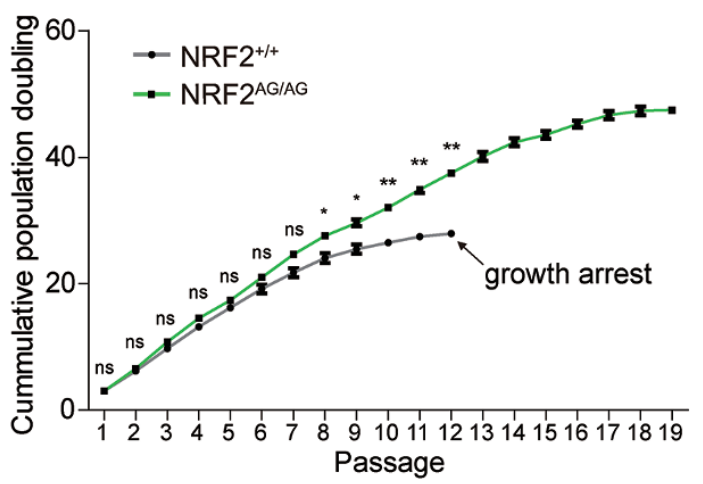

F

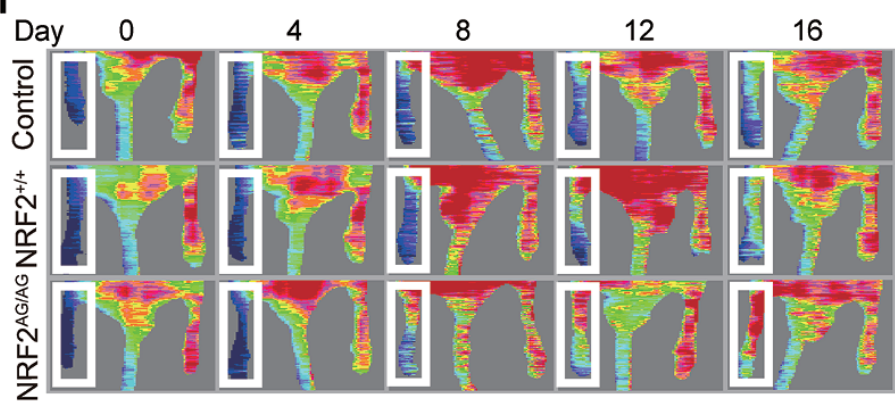

B Cytoplasm
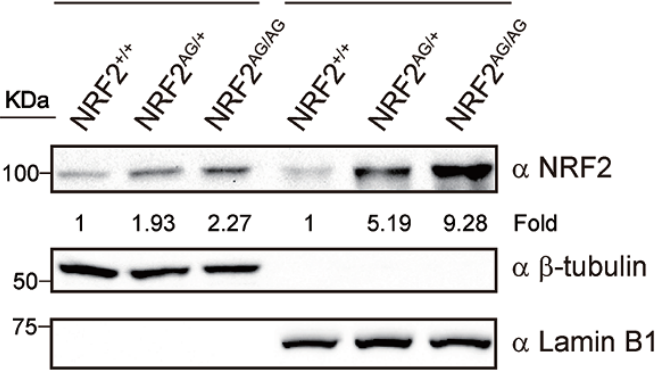

D
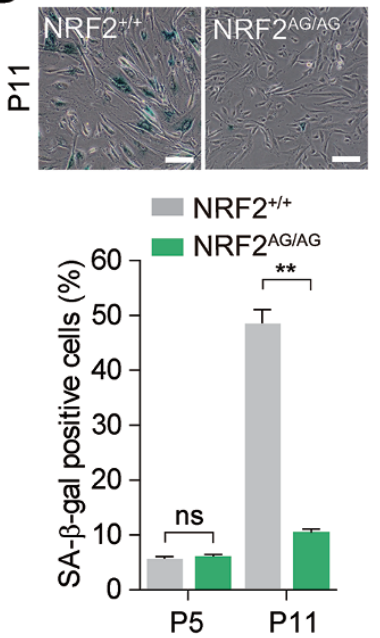

G

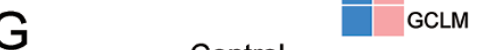

RNA-seq

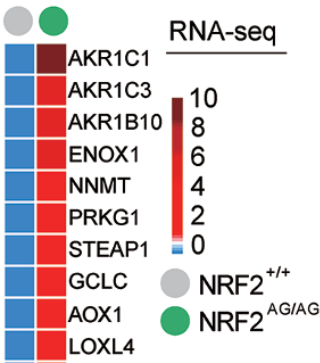

OSGIN1

TXNRD1

ME1

SQRDL

FTL

EPHX1

PTGR1

PGD

NQO1

PLOD2

ASPH

TALDO1

SRXN1

HO-1

FTH1

UGDH

CYB5A

LDHA

GSR

FADS1

FADS3

TXN

G6PD

MICAL2

GCLM

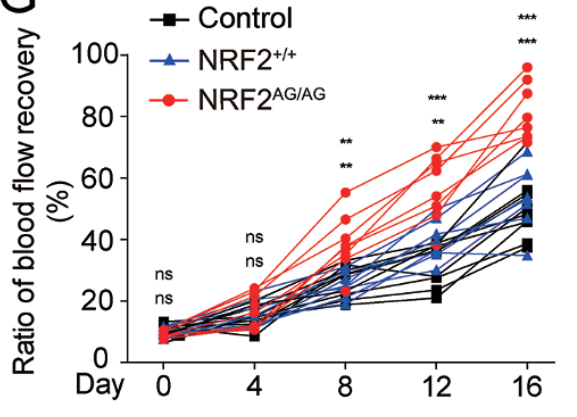

$\mathrm{H}$
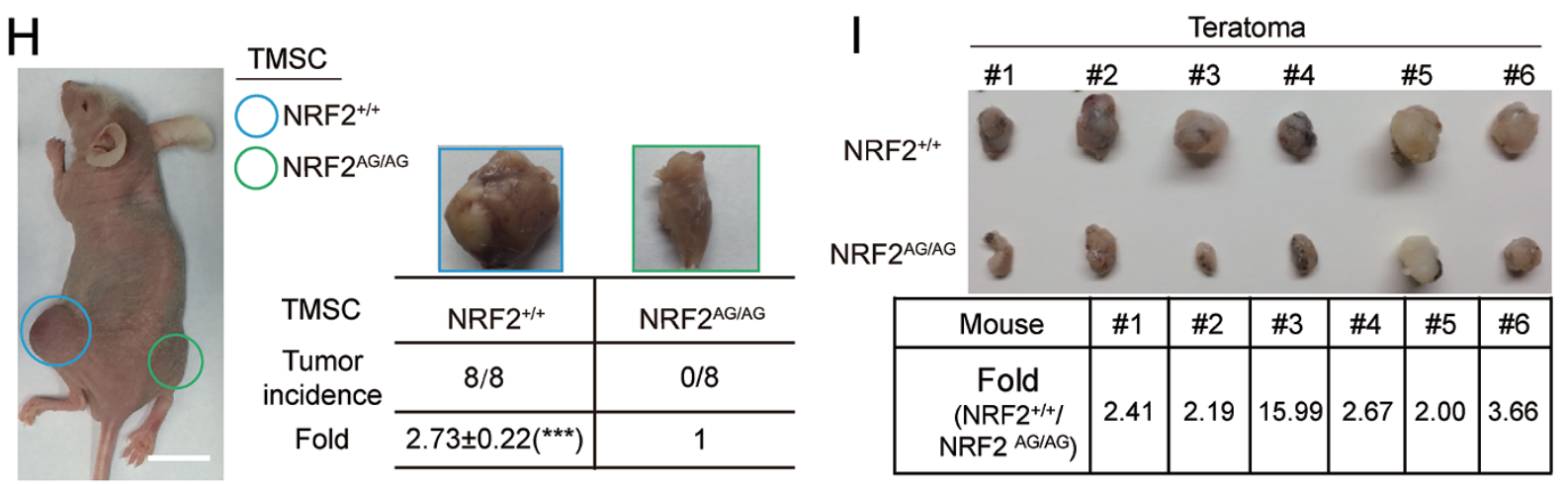

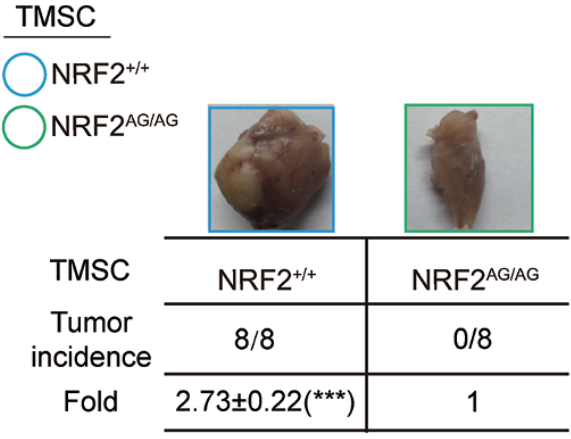

Figure 1 (A) Schematic diagram of gene editing at NRF2 locus and Sanger sequencing results of $N R F 2^{+/+}, N R F 2^{A G /+}$, and $N R F 2^{A G / A G}$ hESCs. (B) Western blot analysis of NRF2 proteins in the cytoplasmic and nuclear extracts of hMSCs. $\beta$-tubulin and Lamin B1 were used as cytoplasmic and nuclear loading controls, respectively. (C) Growth curve showing the accumulative population doubling of hMSCs, $n=3$, ns, not significant, ${ }^{*} P<0.05$, ${ }^{* *} P<0.01$. (D) SA- $\beta$-gal staining of $N R F 2^{+/+}$and $N R F$ - 
NRF2 contents, diminished ROS levels and alleviated accelerated aging phenotypes (Supplementary information, Figure S7).

To understand the molecular mechanism by which genetic manipulation of NRF2 antagonizes cellular senescence in hMSCs, we performed RNA sequencing (RNA-seq) analysis (Supplementary information, Figure S8A-S8D). In P5 hMSCs, 36 upregulated $\left(N R F 2^{A G / A G}\right.$ vs $\left.N R F 2^{+/+}>1.5\right)$ NRF2-responsive cytoprotective genes were identified (Figure 1E). The upregulated genes were involved in stress response, cell viability, and cell proliferation (Supplementary information, Figure S8E), consistent with the stress resistance and improved survival and proliferation observed in $N R F 2^{A G / A G}$ hMSCs. In P11 hMSCs, $\sim 15 \%$ (159/1 090) of the upregulated genes were involved in cell cycle regulation (Supplementary information, Figure S8F), in line with the sustained proliferation of $N R F 2^{A G / A G}$ hMSCs. Expression of over 50 DNA damage repair genes, NE protein-encoding genes and chromosome organization-associated genes was largely maintained in $N R F 2^{A G / A G} \mathrm{hMSCs}$ at P11 (Supplementary information, Figure S9A and S9B), consistent with well-preserved DNA damage repair systems and nuclear architecture in $N R F 2^{A G / A G}$ hMSCs. In addition, transcriptomic analysis revealed less pronounced transcriptional changes in $N R F 2^{A G / A G} \mathrm{hMSCs}$ during passaging (Supplementary information, Figure S9C and S9D).

To determine whether these genetically enhanced stem (GES) cells would confer better survival, engraftment and injury repair in vivo, we first implanted luciferase-labeled hMSCs into the muscle of nude mice $[7,8]$. As expected, compared to WT hMSCs, $N R F 2^{A G / A G}$ hMSCs exhibited slower in vivo decay after transplantation (Supplementary information, Figure S10A). Next, we utilized a well-established hind-limb ischemia murine model to evaluate the in vivo regenerative capacity of hMSCs [9] After ligation of the femoral artery, blood perfusion of the left hind limb was reduced to about 10\% that of the right hind limb in all mice examined (Figure 1F-1G). When compared to mice receiving WT hMSCs, a significantly faster blood flow recovery was observed in mice receiving $N R F 2^{A G / A G}$ hMSCs starting from day 8. In addition, we identified the presence of more human vascular endothelial and smooth muscle cells in $N R F 2^{A G / A G} \mathrm{hMSCs}$-implanted muscles (Figure 1F-1G and Supplementary information, Figure S10B and S10C). These data indicate that edited hMSCs are superior to WT hMSCs in repairing tissue damage in vivo.

Oncogenic transformation of genetically modified cells constitutes a major safety concern [4]. Continuous monitoring for 8 months after $N R F 2^{A G / A G}$ hMSCs transplantation did not indicate any sign of tumor formation in immunodeficient mice (Supplementary information, Figure S11A), demonstrating that tumor susceptibility was not elevated in $N R F 2^{A G / A G}$ hMSCs. However, it is still possible that $N R F 2^{A G / A G}$ hMSCs are susceptible to tumorigenic transformation in the presence of strong oncogenic insults. To examine this possibility, we adopted a reported in vitro MSC transformation system [10] which is capable of transforming hMSCs into cancer cell-like transformed MSCs (TMSCs) (Supplementary information, Figure S11B-S11E). Interestingly, $N R F 2^{A G / A G}$ TMSCs exhibited strikingly compromised in vitro anchorage-independent growth (Supplementary information, Figure S11F and S11G), an important feature of cancer cells characteristic of their transition into a solid tumor. We next evaluated the in vivo tumor forming abilities of the $N R F 2^{A G / A G}$ TMSCs. While palpable tumors in $N R F 2^{+/+}$TMSCs-injected left legs were observed within 6 weeks after the implantation, $N R F 2^{A G / A G}$ TMSCs failed

\footnotetext{
$2^{A G / A G}$ hMSCs. Scale bar, $50 \mu \mathrm{m}$. Data were presented as mean \pm SEM, $n=6$, ns, not significant, ${ }^{* *} P<0.01$. (E) Heatmap of 36 NRF2-responsive cytoprotective genes upregulated in NRF2 $2^{A G / A G} \mathrm{hMSCs}$ at P5 (NRF2 ${ }^{A G / A G}$ vs NRF2 $\left.2^{+/+}>1.5, q<0.05\right)$. All FPKMs of the indicated genes were normalized by the ones in $N R F 2^{+/+}$group and the relative expression level were presented as $\log _{1.5}\left(N R F 2^{A G / A G} / N R F 2^{+/+}\right)$. (F) Representative blood flow imaging of hindlimb ischemic mice injected with PBS (Control), $N R F 2^{+/+}$and NRF2 ${ }^{A G / A G}$ hMSCs (P8). Laser Doppler blood perfusion measurement was performed every 4 days to monitor changes in hindlimb blood flow. The white rectangles indicate the ischemic legs. (G) Blood flow recovery kinetics of each hindlimb ischemic mouse $(n=8)$ after transplantation. Statistical significance was compared between the following: NRF2 ${ }^{A G /}$ ${ }^{A G}$ group vs PBS group (upper); and $N R F 2^{A G / A G}$ group vs $N R F 2^{+/+}$group (lower); ns, not significant, ${ }^{* *} P<0.01,{ }^{* * *} P<0.001 .(\mathrm{H})$ The in vivo tumor forming abilities of the TMSCs. NRF2 ${ }^{+/+}$and $N R F 2^{A G / A G}$ TMSCs were injected into the same immunodeficient mouse in the left and right tibia, respectively, proximal to the knee-joint. While palpable tumors in NRF2 ${ }^{+/} \mathrm{TMSC}^{-i n j e c t e d ~ l e f t}$ legs were detectable within 6 weeks after the implantation, NRF2 ${ }^{A G / A G}$ TMSC failed to form tumors even after 10 weeks. Left, A representative image showing the in vivo tumor formation after TMSCs transplantation. Scale bar, $1 \mathrm{~cm}$. Right, Statistic results of the in vivo tumor-formation of TMSCs. The weight of the left leg was, on average, 2.73-fold higher than that of the right leg. Leg weight data were presented as mean $\pm \mathrm{SD}, n=8,{ }^{* * *} P<0.001$. Leg image and tumor incidence were recorded 10 weeks after transplantation. (I) Images and relative weights of hESC-derived teratomas from 6 transplanted NOD/SCID mice (\#1-6). NRF2 $2^{+/+}$and $N R F 2^{A G / A G}$ hESCs were subcutaneously implanted into both flanks of the same NOD/SCID mouse. Teratoma images were recorded 8 weeks after transplantation.
} 
to form any tumor even after 10 weeks (Figure $1 \mathrm{H}$ and Supplementary information, Figure $\mathrm{S} 11 \mathrm{H}$ ). Genomic copy number variation (CNV) analysis revealed a gained $\mathrm{CNV}$ pattern on chromosome 4 only in $N R F 2^{+/+}$but not in $N R F 2^{A G / A G}$ TMSCs (Supplementary information, Figure S12A), suggesting that genetically activated NRF2 pathway might help safeguard genomic stability against oncogenic insults. RNA-seq and ChIP-qPCR analyses further demonstrated that enhanced endogenous NRF2 selectively activated the expression of specific target genes associated with tumor suppression in TMSCs (Supplementary information, Figure S12B-S12D and Figure S13A-S13C). These data indicate that $N R F 2^{A G / A G}$ hMSCs are refractory to tumorigenic transformation.

Finally, we observed that the implantation of genetically edited hESCs $\left(N R F 2^{A G / A G}\right.$ hESCs) resulted in the generation of much smaller teratomas in vivo (Figure 1I), supporting a possibility that even if residual undifferentiated $N R F 2^{A G / A G}$ hESCs are present in their MSC derivatives, the teratoma-forming ability of these cells are minimalized. This will pose less risk for teratoma formation, which is a major concern for cell replacement therapies using hESC derivatives.

In conclusion, our study provides an experimental proof-of-concept that recoding a single nucleotide within NRF2 gene endows cultured hMSCs with enhanced self-renewal ability, increased stress resistance, delayed cellular senescence and functional decay, better engraftment efficiency and functional regeneration in vivo, as well as greater resistance to oncogenic transformation, and thereby allows for the generation of superior and safer GES cells for regenerative medicine therapies (Supplementary information, Figure S14).

\section{Acknowledgments}

We are grateful to Xiuling $\mathrm{Xu}$, Ying Hao, Xiaoqian Zhang, and Mingming Wei for technical assistance. This work was supported by the National Key R\&D Program of China (2015CB964800, 2017YFA0103300, 2017YFA0102700, 2014CB910503, 2014CB964600), the National High Technology Research and Development Program of China (2015AA020307), CAS (XDA01020312, KJZDEW-TZ-L05, and CXJJ-16M271), the National Natural Science Foundation of China $(81625009,81330008$, 31222039, 81371342, 81300261, 81271266, 81471414, 81422017, 81401159, 31671429, 81601233, 81671377, 31601109, 31601158, 81561138005,31625018 and 81521002), Beijing Natural Science Foundation (7141005 and 5142016), Program of Beijing Munic- ipal Science and Technology Commission (Z151100003915072), the Thousand Young Talents Program of China, and the State Key Laboratory of Stem Cell and Reproductive Biology (2016SRLabKF13). WZ was supported by NIH grants CA158055, CA200673, and CA203834, the V Scholar award, American Cancer Society seed grant, Breast Cancer Research Award and Oberley Award (National Cancer Institute Award P30CA086862) from Holden Comprehensive Cancer Center at the University of Iowa. The Lab of JCIB was supported by UCAM, the G Harold and Leila Y Mathers Charitable Foundation, the Leona M and Harry B Helmsley Charitable Trust (2012-PG-MED002) and the Moxie Foundation.

Jiping Yang ${ }^{1,2,4, *}$, Jingyi $\mathrm{Li}^{1,3,8,9,{ }^{*}}$, Keiichiro Suzuki ${ }^{5,}$, Xiaomeng $\mathrm{Liu}^{3,9}$, Jun $\mathrm{Wu}^{5,6}$, Weiqi Zhang ${ }^{1,2,8}$, Ruotong Ren ${ }^{1,2,8}$, Weizhou Zhang, ${ }^{7}$ Piu Chan ${ }^{8,11}$, Juan Carlos Izpisua Belmonte ${ }^{5}$, Jing $\mathrm{Qu}^{2,4}$, Fuchou Tang ${ }^{3,9,10}$, Guang-Hui Liu ${ }^{1,4,8,11}$

\footnotetext{
${ }^{I}$ National Laboratory of Biomacromolecules, CAS Center for Excellence in Biomacromolecules, Institute of Biophysics, Chinese Academy of Sciences, Beijing 100101, China; ${ }^{2}$ State Key Laboratory of Stem Cell and Reproductive Biology, Institute of Zoology, Chinese Academy of Sciences, Beijing 100101, China; ${ }^{3}$ Beijing Advanced Innovation Center for Genomics (ICG), Ministry of Education Key Laboratory of Cell Proliferation and Differentiation, College of Life Sciences, Peking University, Beijing 100871, China; ${ }^{4}$ University of Chinese Academy of Sciences, Beijing 100049, China, ${ }^{5}$ Gene Expression Laboratory, Salk Institute for Biological Studies, 10010 North Torrey Pines Road, La Jolla, CA 92037, USA; ${ }^{6}$ Universidad Católica San Antonio de Murcia (UCAM) Campus de los Jerónimos, N 135 Guadalupe 30107 Murcia, Spain; ${ }^{7}$ Department of Pathology, Carver College of Medicine, University of Iowa, Iowa City, IA 52242, USA; ${ }^{8}$ National Clinical Research Center for Geriatric Disorders, Xuanwu Hospital of Capital Medical University; ${ }^{9}$ Biomedical Institute for Pioneering Investigation via Convergence, Peking University, Beijing 100871, China; ${ }^{10}$ Peking-Tsinghua Center for Life Sciences, Peking University, Beijing 100871, China:

${ }^{11}$ Beijing Institute for Brain Disorders, Beijing 100069, China

*These three authors contributed equally to this work.

Correspondence: Guang-Hui Liu ${ }^{\mathrm{a}}$, Fuchou Tang ${ }^{\mathrm{b}}$, Jing $\mathrm{Qu}^{\mathrm{c}}$

âE-mail: ghliu@ibp.ac.cn

${ }^{b}$ E-mail: tangfuchou@pku.edu.cn

${ }^{c} E-m a i l:$ qujing@ioz.ac.cn
}

\section{References}

1 Collado M, Blasco MA, Serrano M. Cell 2007; 130:223-233.

2 Campisi J. Annu Rev Physiology 2013; 75:685-705.

3 Hentze H, Graichen R, Colman A. Trends Biotechnol 2007; 25:24-32.

4 Wang Y, Han ZB, Song YP, et al. Stem Cells Int 2012; 2012:65204.

5 Hockemeyer D, Jaenisch R. Cell Stem Cell 2016; 18:573-586.

6 Yamanaka S. Cell Stem Cell 2012; 10:678-684.

7 Zhang W, Li J, Suzuki K, et al. Science 2015; 348:1160-1163.

8 Pan H, Guan D, Liu X, et al. Cell Res 2016; 26:190-205.

9 Lian Q, Zhang Y, Zhang J, et al. Circulation 2010; 121:1113-1123.

10 Scaffidi P, Misteli T. Nat Cell Biol 2011; 13:1051-1061.

(Supplementary information is linked to the online version of the paper on the Cell Research website.) 